\title{
OPEN High 1-year risk of stroke in patients with hepatocellular carcinoma: a nationwide registry-based cohort study
}

\begin{abstract}
Jin-Yi Hsu ${ }^{1,2}$, Peter Pin-Sung Liu1 ${ }^{1}$ An-Bang Liu ${ }^{2,3}$, Huei-Kai Huang ${ }^{2,4,5}$ \& Ching-Hui Loh ${ }^{1,2,4 \bowtie ~}$
Patients with hepatocellular carcinoma (HCC) might be more vulnerable to develop stroke than other cancer patients because of HCC-associated coagulation dysfunction. However, limited studies have investigated the relationship between HCC and stroke. This nationwide population-based cohort study enrolled all patients with HCC diagnosed between 2011 and 2015 from the Taiwan Cancer Registry and Taiwan National Health Insurance Research Database; an age- and sex-matched cohort without cancer was included. The primary outcome was the 1-year risk for first-ever stroke after the index date. The Fine and Gray competing risk regression model was used to estimate the 1-year stroke risk with adjusted hazard ratios (aHRs). After propensity score matching, each cohort has 18,506 patients with similar baseline characteristics. Compared with the cancer-free cohort, the aHRs in the HCC cohort for overall, ischemic, and hemorrhagic strokes were 1.59 [95\% confidence interval ( $\mathrm{Cl}), 1.35-1.88], 1.38$ [95\% Cl, 1.15-1.65], and 2.62 [95\% Cl, 1.79-3.84], respectively. On subgroup analysis, HCC patients without cirrhosis, those with stage 3 or 4 cancer had a higher stroke risk than cancer-free cohort. Therefore, stroke prevention should be considered in patients with HCC, especially in those without cirrhosis and with stage 3 or 4 cancer.
\end{abstract}

Hepatocellular carcinoma (HCC) is one of the most common type of cancer worldwide ${ }^{1}$. Although HCC is historically lethal, the 5 -year survival rate has increased to nearly $50 \%$ because of better surveillance strategies and surgical management of $\mathrm{HCC}^{2}$. Previous studies have suggested that cancer is a potential risk factor for stroke; the risk of stroke varies depending on the cancer type, cancer stage, and duration after the cancer diagnosis ${ }^{3-5}$. The main mechanism for cancer-related stroke is cancer-associated hypercoagulability resulting from an abundance of hypercoagulable materials, tissue factors, or mucins secreted by cancer cells ${ }^{6,7}$. Moreover, chronic disseminated coagulopathy promotes bleeding tendency because of the extended consumption of coagulation factors ${ }^{8}$. In addition to cancer-associated coagulopathy, patients with HCC often have coagulation dysfunction because of cirrhosis or accumulation of malignant tumor cells in the liver ${ }^{9,10}$. With regards to the relationship between stroke and cancer, HCC should be considered as an important risk factor for ischemic stroke or hemorrhagic stroke due to coagulation dysfunction. Some studies revealed that HCC cells might be associated with systemic thrombotic and hemorrhagic processes ${ }^{10,11}$. However, to date, there is little evidence to clarify the association between HCC and stroke, both ischemic and hemorrhagic. Only one previous observational study analyzed the association between HCC and stroke in their subgroup analyses. However, this study was not specifically designed to evaluate the association between the risk of stroke and HCC, and thus did not adjust for important confounders related to the risk of stroke in patients with HCC and did not obtain data on cancer stages ${ }^{12}$.

We conducted a nationwide, whole-population, registry-based cohort study to clarify the relationship between HCC and risk of stroke. We hypothesize that patients with HCC might have a higher 1-year risk of stroke compared with cancer-free individuals.

\footnotetext{
${ }^{1}$ Center for Aging and Health, Hualien Tzu Chi Hospital, Buddhist Tzu Chi Medical Foundation, Hualien, Taiwan, No. 707, Sec. 3, Chung Yang Rd., 97002. ${ }^{2}$ School of Medicine, Tzu Chi University, Hualien, Taiwan. ${ }^{3}$ Department of Neurology, Hualien Tzu Chi Hospital, Buddhist Tzu Chi Medical Foundation, Hualien, Taiwan. ${ }^{4}$ Department of Family Medicine, Hualien Tzu Chi Hospital, Buddhist Tzu Chi Medical Foundation, Hualien, No. 707, Sec. 3, Chung Yang Rd., 97002, Taiwan. ${ }^{5}$ Department of Medical Research, Hualien Tzu Chi Hospital, Buddhist Tzu Chi Medical Foundation, Hualien, Taiwan. ${ }^{\bowtie}$ email: drhkhuang@gmail.com; twdoc1960@gmail.com
} 


\begin{tabular}{|c|c|c|c|}
\hline \multirow[b]{2}{*}{ Characteristics } & HCC cohort & Cancer-free cohort & Standardized \\
\hline & $N=18,506$ & $N=18,506$ & Difference \\
\hline Age $^{\mathrm{a}}$ & $63.2(12.1)$ & $62.8(11.8)$ & 0.029 \\
\hline Male & $12,813(69.2)$ & $13,314(71.9)$ & 0.059 \\
\hline \multicolumn{4}{|l|}{ Income (NTD) } \\
\hline Dependence & $3780(20.4)$ & $3554(19.2)$ & 0.031 \\
\hline $15,800-29,999$ & $8725(47.2)$ & $9243(50.0)$ & 0.056 \\
\hline $30,000-44,999$ & $3511(19.0)$ & $3464(18.7)$ & 0.006 \\
\hline 45,000 and above & $2490(13.5)$ & $2245(12.1)$ & 0.040 \\
\hline Charlson comorbidity index ${ }^{a}$ & $2.8(2.7)$ & $2.5(2.2)$ & 0.127 \\
\hline \multicolumn{4}{|l|}{ Comorbidities } \\
\hline Hypertension & $8667(46.8)$ & $8570(46.3)$ & 0.010 \\
\hline Diabetes mellitus & $5257(28.4)$ & $5181(28.0)$ & 0.009 \\
\hline Dyslipidemia & $5233(28.3)$ & $4759(25.7)$ & 0.058 \\
\hline Atrial fibrillation & $401(2.2)$ & $302(1.6)$ & 0.040 \\
\hline Valvular heart disease & $135(0.7)$ & $113(0.6)$ & 0.015 \\
\hline Congestive heart failure & $758(4.1)$ & $624(3.4)$ & 0.039 \\
\hline Coronary artery disease & $2745(14.8)$ & $2462(13.3)$ & 0.044 \\
\hline Peripheral arterial occlusion disease & $384(2.1)$ & $278(1.5)$ & 0.044 \\
\hline Cirrhosis & $5946(32.1)$ & $5935(32.1)$ & 0.001 \\
\hline Chronic kidney disease & $975(5.3)$ & $904(4.9)$ & 0.018 \\
\hline Major gastrointestinal bleeding & $175(1.0)$ & $216(1.2)$ & 0.022 \\
\hline \multicolumn{4}{|l|}{ Medication use $^{\text {b }}$} \\
\hline Antithrombotic therapy $^{c}$ & $2293(12.4)$ & $1937(10.5)$ & 0.060 \\
\hline Anticoagulant & $231(1.3)$ & $176(1.0)$ & 0.029 \\
\hline Antiplatelet & $2101(11.4)$ & $1790(9.7)$ & 0.055 \\
\hline
\end{tabular}

Table 1. Baseline characteristics in patients with hepatocellular carcinoma and the cancer-free cohort after propensity score matching. Data are expressed as n (\%) unless otherwise indicated. NTD New Taiwan dollar, HCC hepatocellular carcinoma, SD standard deviation. ${ }^{a}$ Expressed as the mean (SD). ${ }^{\mathrm{b}}$ Medication use means a drug prescription for more than 30 days during the observation period. ${ }^{\mathrm{c}}$ Antithrombotic therapy means either anticoagulant or antiplatelet use.

\section{Results}

Patient characteristics. This study included 33,468 patients with HCC and 66,936 patients with ageand sex-matching as cancer-free individuals. Compared with cancer-free individuals, patients with HCC had a higher Charlson comorbidity index, a higher prevalence of diabetes mellitus, cirrhosis, and a history of major gastrointestinal bleeding (Table S1). After propensity score matching, each cohort had 18,506 patients and the characteristics in each cohort were similar with all standardized difference values $<0.2$ (Table 1 ).

Risk of stroke. The HCC cohort had 361 patients diagnosed with stroke and the cancer-free cohort had 236 patients diagnosed with stroke 1 year after the index date. The HCC cohort had a higher cumulative incidence of developing stroke overall (Gray's test, $\mathrm{p}<0.001$; Fig. 1A), as well as ischemic (Gray's test, $\mathrm{p}=0.001$; Fig. 1B) and hemorrhagic (Gray's test, $\mathrm{p}<0.001$; Fig. 1C) stroke subtypes, than the cancer-free cohort. The multivariable regression models revealed that HCC was associated with a higher risk of developing stroke (adjusted hazard ratio [aHR], 1.59; 95\% CI, 1.35-1.88). The sub-analyses further revealed that the risks of both ischemic stroke (aHR, 1.38; 95\% CI, 1.15-1.65) and hemorrhagic stroke (aHR, 2.62; 95\% CI, 1.79-3.84) (Table 2) were higher in the HCC cohort compared with those in the cancer-free cohort.

Subgroup analyses for cirrhosis, cancer stages, and cancer treatment. We analyzed the association between HCC and risk of stroke after sub-grouping the HCC cohort according to the presence of cirrhosis, cancer stage, and cancer treatment. The HCC patients in each subgroup were compared with their matched cancer-free individuals. Compared with the cancer-free cohort, HCC patients without cirrhosis, and those with stage 3, stage 4, or an unknown stage cancer had a higher risk of stroke (Table 3). HCC patients also had higher risk of stroke than the cancer-free individuals regardless of receiving cancer treatment. The subgroups analysis for both ischemic stroke (Table S2) and hemorrhagic stroke (Table S3) disclosed similar findings.

Sensitivity analyses. The sensitivity analysis, involving all study participants without matching, revealed similar findings as the results after propensity score matching. The HCC cohort still had a higher risk of stroke overall (aHR, 1.68; 95\% CI, 1.46-1.94) as well as a higher risk of ischemic stroke (aHR, 1.45; 95\% CI, 1.23-1.71) and hemorrhagic stroke (aHR, 2.73; 95\% CI, 2.03-3.66) compared with non-cancer cohort (Table 4). 
A Cumulative incidence curves of stroke

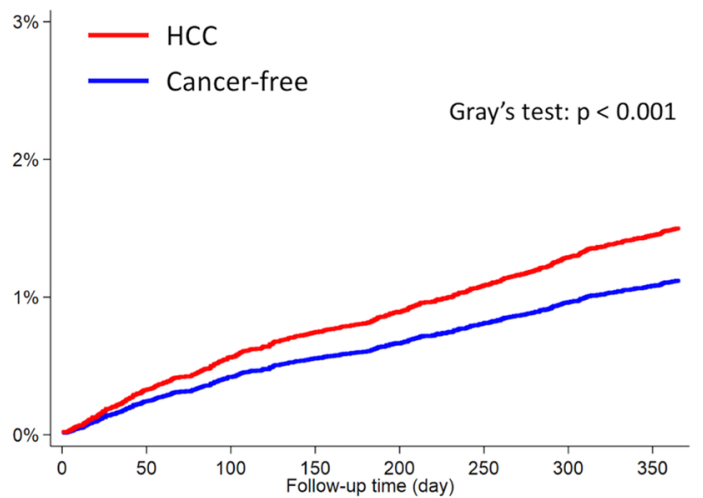

B Cumulative incidence curves of ischemic stroke

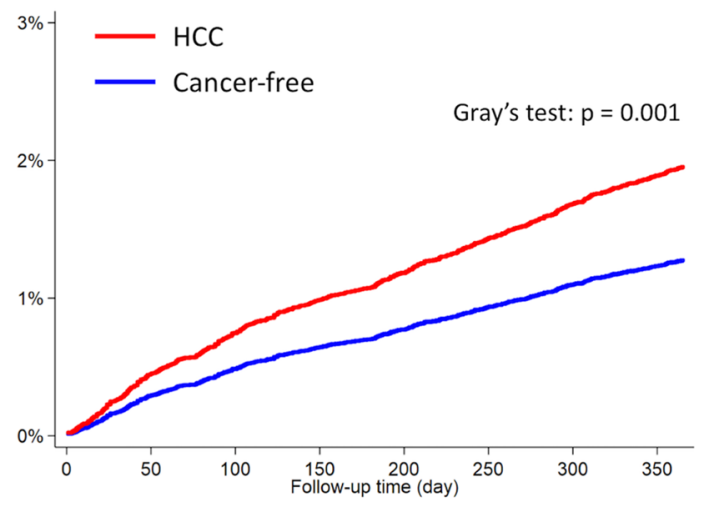

C Cumulative incidence curves of hemorrhagic stroke

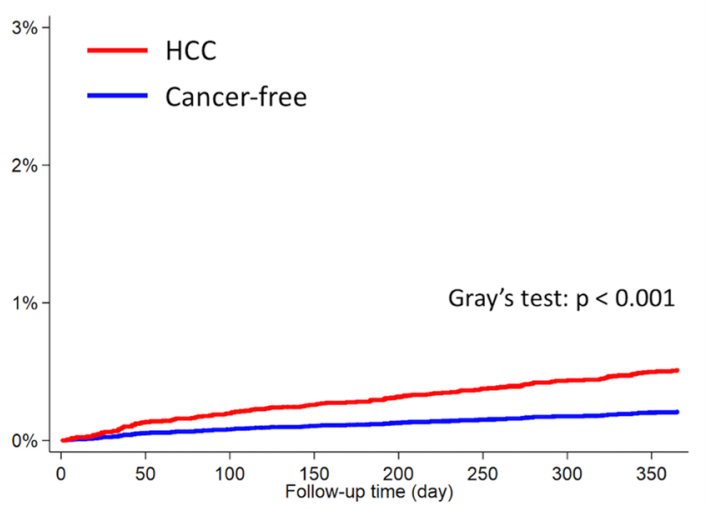

Figure 1. Cumulative incidence curves of stroke in patients with HCC and cancer-free individuals. (A) Stroke overall; (B) ischemic stroke; (C) hemorrhagic stroke. HCC hepatocellular carcinoma.

\begin{tabular}{|c|c|c|c|c|c|c|}
\hline \multirow[b]{2}{*}{ Outcome } & \multicolumn{2}{|c|}{ HCC cohort } & \multicolumn{2}{|c|}{ Cancer-free cohort } & \multirow[b]{2}{*}{$\mathrm{aHR}^{\mathrm{b}}(95 \% \mathrm{CI})$} & \multirow[b]{2}{*}{$p$ value } \\
\hline & Event & $\mathrm{IR}^{\mathrm{a}}$ & Event & $\mathbf{I R}^{\mathrm{a}}$ & & \\
\hline All stroke & 361 & 5.68 & 236 & 3.52 & $1.59(1.35-1.88)$ & $<0.001$ \\
\hline Ischemic stroke & 277 & 4.36 & 207 & 3.09 & $1.38(1.15-1.65)$ & 0.001 \\
\hline Hemorrhagic stroke & 94 & 1.48 & 38 & 0.57 & $2.62(1.79-3.84)$ & $<0.001$ \\
\hline
\end{tabular}

Table 2. Risk of stroke in patients with hepatocellular carcinoma compared with the cancer-free cohort after propensity score matching. ${ }^{a}$ IR per 1000 person-years. ${ }^{\text {b}}$ The hazard ratios were calculated using a multivariable Cox proportional hazards regression model with adjustments for income, CCI, comorbidities, and medication use listed in Table 1. aHR adjusted hazard ratio, CI confidence interval, HCC hepatocellular carcinoma, IR incidence rate, CCI: Charlson comorbidity index.

\section{Discussion}

This study demonstrates that patients with HCC have a higher risk of stroke compared with cancer-free individuals. Patients with HCC have a 59\% higher risk of all stroke types, with a $38 \%$ higher risk of ischemic stroke and a 162\% higher risk of hemorrhagic stroke compared with cancer-free individuals. The stroke risk varied based on presence of cirrhosis and different cancer stages; patients without cirrhosis and those with stage 3 or stage 4 cancer had a higher risk of stroke compared with cancer-free individuals. To our knowledge, this is the largest cohort study to specifically investigate the association between HCC and stroke, and the first cohort shows that HCC is associated with higher risk of ischemic stroke.

The association between cancer and stroke has been reported in many common cancers, such as lung cancer $^{4,12,20}$, breast cancer ${ }^{4,12,20}$, colorectal cancer ${ }^{4,12,20}$, prostate cancer ${ }^{4,12,20}$, and stomach cancer ${ }^{4,12}$. HCC is one of the common types of cancer worldwide, but few reports discuss the relationship between HCC and stroke ${ }^{12}$. HCC has high mortality rate; this poor statistic has prevented research concerning the association between HCC and stroke. In Taiwan, the 5-year survival rate of HCC is higher than $50 \%^{2}$; correspondingly, this difficult topic could further elucidate through this nationwide cancer registry database investigation. These results might raise questions about whether stroke prevention might be beneficial in patients with HCC when patients have longer expected survival times. 


\begin{tabular}{|c|c|c|c|}
\hline & $\mathrm{aHR}^{\mathrm{a}}$ & $95 \%$ CI & p value \\
\hline \multicolumn{4}{|l|}{ Cirrhosis } \\
\hline Yes & 1.24 & $0.87-1.75$ & 0.229 \\
\hline No & 1.73 & $1.42-2.09$ & $<0.001$ \\
\hline \multicolumn{4}{|c|}{ Cancer stage } \\
\hline 1 & 0.93 & $0.69-1.26$ & 0.650 \\
\hline 2 & 0.97 & \begin{tabular}{|l|}
$0.68-1.39$ \\
\end{tabular} & 0.878 \\
\hline 3 & 2.22 & $1.51-3.27$ & $<0.001$ \\
\hline 4 & 4.90 & \begin{tabular}{|l|}
$3.00-7.99$ \\
\end{tabular} & $<0.001$ \\
\hline Unknown & 2.07 & $1.33-3.22$ & 0.002 \\
\hline \multicolumn{4}{|c|}{ Cancer treatment ${ }^{b}$} \\
\hline Yes & 1.49 & \begin{tabular}{|l|}
$1.23-1.80$ \\
\end{tabular} & $<0.001$ \\
\hline No & 1.98 & $1.38-2.84$ & $<0.001$ \\
\hline
\end{tabular}

Table 3. Subgroup analyses to assess risk of stroke in patients with hepatocellular carcinoma compared with the cancer-free individuals according to the presence of cirrhosis, cancer stages, and cancer treatment. $a H R$ adjusted hazard ratio, $C I$ confidence interval, $C C I$ Charlson comorbidity index. ${ }^{\text {a }}$ The hazard ratios were calculated using a multivariable Cox proportional hazards regression model with adjustments for income, CCI, comorbidities, and medication use listed in Table 1. Cancer-free controls comprised the reference group for analysis, and the information for the cancer-free reference group is not provided in the table. ${ }^{\mathrm{b}}$ Cancer treatment means patients had either surgical therapy, chemotherapy, or radiation therapy.

\begin{tabular}{|c|c|c|c|c|c|c|}
\hline \multirow[b]{2}{*}{ Outcome } & \multicolumn{2}{|c|}{ HCC cohort } & \multicolumn{2}{|c|}{ Cancer-free cohort } & \multirow[b]{2}{*}{$\mathrm{aHR}^{\mathrm{b}}(95 \% \mathrm{CI})$} & \multirow[b]{2}{*}{$p$ value } \\
\hline & Event & $\mathrm{IR}^{\mathrm{a}}$ & Event & $\mathrm{IR}^{\mathrm{a}}$ & & \\
\hline All stroke & 603 & 5.21 & 664 & 2.73 & $1.68(1.46-1.94)$ & $<0.001$ \\
\hline Ischemic stroke & 445 & 3.85 & 577 & 2.38 & $1.45(1.23-1.71)$ & $<0.001$ \\
\hline Hemorrhagic stroke & 174 & 1.50 & 114 & 0.47 & $2.73(2.03-3.66)$ & $<0.001$ \\
\hline
\end{tabular}

Table 4. Risk of stroke in patients with hepatocellular carcinoma compared with the cancer-free cohort without propensity score matching. $a H R$ adjusted hazard ratio, CI confidence interval, CCI Charlson comorbidity index, HCC hepatocellular carcinoma, IR incidence rate. ${ }^{a} I R$ per 1000 person-years. ${ }^{\text {b}}$ The hazard ratios were calculated using a multivariable Cox proportional hazards regression model with adjustments for income, CCI, comorbidities, and medication use listed in Table 1.

Previous studies investigating the association between HCC and stroke were typically only part of a subgroup analysis. Zöller et. al examined a cohort of patients with various cancers to investigate the association between the risk of various types of stroke and cancer ${ }^{12}$. In their subgroup analysis, patients with HCC only showed a higher risk of hemorrhagic stroke in the first 6 months after being diagnosed with HCC than cancer-free individuals, but there was a similar risk of ischemic stroke in patients with HCC and cancer-free individuals. In their analysis, they only considered conventional stroke risk factors as covariates in their multivariable analysis, such as age, hypertension, or diabetes mellitus. However, they had less emphasis on risk factors specific for patients with HCC, such as cirrhosis of the liver ${ }^{9}$, chronic kidney disease, or other factors related to bleeding tendency. Likewise, the association between stroke risk and various stages of cancer has been mentioned with respect to other cancers ${ }^{4}$, but there have been no reports concerning patients with HCC. Thus, our study only included patients with HCC and analyzed the relationship between the risk of stroke and HCC, considering stroke risk factors specific for patients with HCC and various HCC stages.

An important question for patients with $\mathrm{HCC}$ is whether they have adequate preserved liver function, even after surgical resection ${ }^{2}$. Although most patients with HCC often have impaired liver function due to cirrhosis, the proportion of noncirrhotic patients with HCC is also increasing because of increased surveillance of HCC in carriers of viral hepatitis and the rising incidence of non-alcoholic fatty liver disease ${ }^{2,21}$. Patients without cirrhosis often had better liver function and 5-year survival rates ${ }^{22}$; thus, they are likely to benefit most from prevention of cancer-associated complications, including stroke. Management for stroke prevention, either by antiplatelet or anticoagulant therapy, might cause major bleeding events such as gastrointestinal bleeding or intracranial hemorrhage ${ }^{23}$. These complications might be controllable in patients with preserved liver function. Our study indicates that patients without cirrhosis had a higher risk of stroke compared with cancer-free individuals; therefore, use of antiplatelets or anticoagulants might confer additional benefits in these patients for stroke prevention, especially during the first year after being diagnosed with HCC. Further studies are warranted to guide clinicians on how to balance the benefits of stroke prevention and the risk of major bleeding events in patients with HCC, especially those with preserved liver function.

This study also revealed that patients with advanced stages of HCC had a higher risk of stroke compared with cancer-free individuals. This manifestation was similar to other cancers mentioned in previous studies ${ }^{4}$. 
The positive relationship between the cancer stage and the amount of procoagulant materials secreted by cancer cells have been reported ${ }^{24}$, perhaps indicating why patients with advanced cancer were associated with a higher risk of stroke. Aside from stroke, portal vein thrombosis is a common cancer-associated complication in patients with HCC. Currently, while routine prophylactic anticoagulants are not recommended for every patient with HCC, personalized prophylactic anticoagulant use for portal vein thrombosis is recommended ${ }^{10}$. Patients with advanced stage HCC might obtain additional stroke prevention benefits when using prophylactic anticoagulants for portal vein thrombosis. Further studies are needed to assess whether prophylactic anticoagulants are advantageous for either portal vein thrombosis or stroke in patients with advanced stage HCC.

The present study has some limitations. First, not all stroke-related factors were adjusted. The administrative database did not provide all conventional stroke factors, thus information regarding body mass index, a history of smoking or drinking, diet, and physical habits were missing; these characteristics might be confounding risk factors for stroke in patients with HCC. Second, the database did not provide detailed laboratory data; hence, it was difficult to estimate the function of liver and coagulation in patients with HCC. Although we tried to balance the difference between patients with HCC and cancer-free individuals by a diagnosis of cirrhosis of the liver, chronic kidney disease, and major gastrointestinal bleeding, the liver status and coagulation function might be potential cofounding factors for stroke.

In conclusion, patients with HCC have a higher 1-year risk of stroke than those without cancer. In the era of increasing survival rate of patients with HCC, strategies for stroke prevention must be actively considered in these patients, especially in those without cirrhosis and those with stage 3 or stage 4 cancer.

\section{Methods}

Study design and data sources. The Taiwan Cancer registry (TCR) and the Taiwan National Health Insurance Research Database (NHIRD) were utilized to conduct a real-world, nationwide, registry-based cohort study. The TCR comprises nearly $90 \%$ of all patients with cancer in Taiwan and contains detailed information related to cancer, such as the staging, treatment, and specific dates of each procedure ${ }^{13,14}$. To obtain healthcare information from patients with cancer, identical encrypted codes were linked with the NHIRD from all 23.6 million enrollees. This TCR-NHIRD composite database provides comprehensive healthcare information, including all hospitalizations, emergency services, outpatient visits, and detailed medication prescription data. Furthermore, patients without cancer in the NHIRD were included as a cancer-free cohort. Both the NHIRD and TCR are maintained by the Health and Welfare Data Science Center of the Ministry of Health and Welfare in Taiwan ${ }^{15}$. The diagnostic and procedure codes of interest were the International Classification of Diseases, Ninth Revision, Clinical Modification (ICD-9-CM), before 2015, and the International Classification of Diseases, Tenth Revision, Clinical Modification (ICD-10-CM), after 2016. Additionally, the diagnostic codes for cancer types were based on the ICD for Oncology, Third Edition site recode classification (ICD-Oncology-3) in the TCR.

Study population. Patients with HCC who were diagnosed with primary HCC, with an ICD-Oncology-3 C22 code in the TCR, from January 1, 2011, to December 31, 2015 were included. In patients with multiple primary cancers, only patients who had a first cancer diagnosis of HCC were recruited. A cancer-free cohort was also incorporated into the study; thus, each patient with HCC was matched to 2 age- and sex-matched patients without cancer from the TCR-NHIRD composite database.

Patients younger than 20 years old, those without complete basic information, those who were unable to be age- and sex-matched, or those having primary outcomes before a cancer diagnosis were excluded. Moreover, patients who died within 180 days of the cancer diagnosis were also excluded because different medical care policies might exist in terminal cancer patients with a shorter life expectancy.

Outcomes. The date of the cancer diagnosis was defined as the index date in the HCC group, and identical dates were set as the index dates in the matched cancer-free individuals. The primary outcome was a composite outcome of either ischemic or hemorrhagic stroke, defined as any inpatient diagnosis of stroke with brain imaging examination. Previous manuscripts have validated these diagnostic criteria to clarify these outcomes in the Taiwan NHIRD ${ }^{16,17}$. An ischemic stroke diagnosis was defined as an inpatient diagnosis of ICD-9-CM codes 433, 434 , and 436 or ICD-10-CM codes I63 and I67.89; a hemorrhagic stroke diagnosis was defined as an inpatient diagnosis with ICD-9-CM codes 430 and 431 or ICD-10-CM I60 and I61. Individuals were followed from the index date until the occurrence of stroke, either ischemic or hemorrhagic, or death, or until 1 year after the index date. In addition to the risk of stroke overall, we also analyzed the ischemic and hemorrhagic stroke separately as individual outcomes. The risk of stroke in patients with HCC was examined compared with cancer-free cohort. To investigate whether the risk of stroke in patients with HCC varied by the presence of cirrhosis, different cancer stages, or having cancer treatment, subgroup analyses were conducted based on these factors.

Covariates. All comorbidities listing in Table 1 were defined as a discharge diagnosis or a diagnosis that was confirmed at least twice in an outpatient department before the index date, based diagnostic codes and procedure code. Charlson comorbidity index was calculated depending on baseline comorbidities; they represent the complexity of comorbidities in each patient ${ }^{18}$. Medication use related to stroke prevention was evaluated after the index date. Medication use was defined as at least 30 days of exposure to each drug during the study period. In patients with HCC, the cancer stage depended on pathological staging unless they had not received further curative operations; in that case, clinical staging was used. Cancer treatment included surgical therapy, chemotherapy, and radiation therapy. 
Sensitivity analysis. Since only part of the population was selected for analysis, there was a possibility of bias when analyzing data with propensity score matching. In order to validate the findings, sensitivity analyses were conducted including all eligible patients without matching; multivariable Fine and Gray competing risk regression models were used to adjust all covariates listed in Table 1.

Statistical analysis. A 1:1 propensity score matching was performed using age, sex, income, Charlson comorbidity index, and comorbidities as listed in Table 1. Propensity score matching utilized a nearest-neighbor matching algorithm without replacement and used a caliper width equal to 0.2 of a standard deviation of the propensity score logit value. Standardized difference was used to assess the difference in baseline characteristics between groups, and a value of less than 0.2 was considered negligible. Regarding higher mortality among patients with HCC, a cumulative incidence function with death as a competing event was employed. The Gray's test was utilized to examine the differences between cumulative incidence curves. Multivariable Fine and Gray competing risk regression models were used to measure the hazard ratios and corresponding $95 \%$ confidence intervals (CIs) for stroke with death as a competing event ${ }^{19}$. When analyzing ischemic stroke as an individual outcome, the event of hemorrhagic stroke was also considered as a competing risk, and vice versa. Age, sex, income, Charlson comorbidity index, comorbidities, and medication use related to stroke prevention were included and adjusted in the multivariable regression models. Statistical significance was defined as a two-tailed probability value $<0.05$. All statistical analyses were performed using SAS, version 9.4 (SAS Institute Inc., Cary, NC) and Stata, version 14.0 (Stata Corporation, College Station, TX).

Ethical approval. Our study were in accordance with the 1964 Helsinki declaration and its later amendments or comparable ethical standards, and thus this study was approved by the Institutional Review Board of Hualien Tzu Chi Hospital (IRB-107-06C).

Informed consent. The Institutional Review Board agreed that informed consent could be waived because these database are encrypted databases.

\section{Data availability}

The TCR and Taiwan NHIRD are an encrypted database that is regulated and maintained by the Health and Welfare Data Science Center at the Ministry of Health and Welfare in Taiwan. Therefore, the data set cannot be available publicly. Researchers interested in analyzing this data set can provide a formal application to the Taiwan Ministry of Health and Welfare to request access (website: https://dep.mohw.gov.tw/DOS/cp-2516-3591-113. html).

Received: 12 July 2020; Accepted: 19 February 2021

Published online: 17 May 2021

\section{References}

1. Villanueva, A. Hepatocellular carcinoma. N. Engl. J. Med. 380, 1450-1462 (2019).

2. Yang, J. D. et al. A global view of hepatocellular carcinoma: Trends, risk, prevention and management. Nat. Rev. Gastroenterol. Hepatol. 16, 589-604 (2019).

3. Velander, A. J., DeAngelis, L. M. \& Navi, B. B. Intracranial hemorrhage in patients with cancer. Curr. Atheroscler. Rep. 14, 373-381 (2012).

4. Navi, B. B. et al. Risk of arterial thromboembolism in patients with cancer. J. Am. Coll. Cardiol. 70, 926-938 (2017).

5. Razak, N. B. A., Jones, G., Bhandari, M., Berndt, M. C. \& Metharom, P. Cancer-associated thrombosis: An overview of mechanisms, risk factors, and treatment. Cancers (Basel). 10, 1-21 (2018).

6. Schwarzbach, C. J. et al. Stroke and cancer: The importance of cancer-associated hypercoagulation as a possible stroke etiology. Stroke 43, 3029-3034 (2012).

7. Varki, A. Trousseau's syndrome: Multiple definitions and multiple mechanisms. Blood 110, 1723-1729 (2007).

8. Levi, M. Management of cancer-associated disseminated intravascular coagulation. Thromb. Res. 140, S66-S70 (2016).

9. Tripodi, A. \& Mannucci, P. M. The coagulopathy of chronic liver disease. N. Engl. J. Med. 365, 147-156 (2011).

10. Zanetto, A. et al. Cancer-associated thrombosis in cirrhotic patients with hepatocellular carcinoma. Cancers (Basel). 10, 1-19 (2018).

11. Samonakis, D. N. et al. Hypercoagulable states in patients with hepatocellular carcinoma. Dig. Dis. Sci. 49, 854-858 (2004).

12. Zöller, B., Ji, J., Sundquist, J. \& Sundquist, K. Risk of haemorrhagic and ischaemic stroke in patients with cancer: A nationwide follow-up study from Sweden. Eur. J. Cancer 48, 1875-1883 (2012).

13. Chiang, C. J. et al. Quality assessment and improvement of nationwide cancer registration system in Taiwan: A review. Jpn. J. Clin. Oncol. 45, 291-296 (2015).

14. Hsieh, C.-Y. et al. Taiwan's National Health Insurance Research Database: Past and future. Clin. Epidemiol. 11, 349-358 (2019).

15. Lin, L., Warren-Gash, C., Smeeth, L. \& Chen, P.-C. Data resource profile: The National Health Insurance Research Database (NHIRD). Epidemiol. Health 40, e2018062-e2018070 (2018).

16. Hsieh, C.-Y., Chen, C.-H., Li, C.-Y. \& Lai, M.-L. Validating the diagnosis of acute ischemic stroke in a National Health Insurance claims database. J. Formos. Med. Assoc. 114, 254-259 (2015).

17. Cheng, C.-L., Kao, Y.-H.Y., Lin, S.-J., Lee, C.-H. \& Lai, M. L. Validation of the National Health Insurance Research Database with ischemic stroke cases in Taiwan. Pharmacoepidemiol. Drug Saf. 20, 236-242 (2011).

18. Charlson, M. E., Pompei, P., Ales, K. L. \& MacKenzie, C. R. A new method of classifying prognostic comorbidity in longitudinal studies: Development and validation. J. Chronic Dis. 40, 373-383 (1987)

19. Fine, J. P. \& Gray, R. J. A proportional hazards model for the subdistribution of a competing risk. J. Am. Stat. Assoc. 94, 496-509 (1999).

20. Navi, B. B. et al. Association between incident cancer and subsequent stroke. Ann. Neurol. 77, 291-300 (2015)

21. Hester, D., Golabi, P., Paik, J., Younossi, I., Mishra, A., Younossi, Z. M. Among Medicare Patients With Hepatocellular Carcinoma, Non-alcoholic Fatty Liver Disease is the Most Common Etiology and Cause of Mortality. J Clin Gastroenterol. 54(5), 459-467 (2020). 
22. Schütte, K. et al. Characterization and prognosis of patients with hepatocellular carcinoma (HCC) in the non-cirrhotic liver. $B M C$ Gastroenterol. 14, 1-10 (2014).

23. Qamar, A., Vaduganathan, M., Greenberger, N. J. \& Giugliano, R. P. Oral anticoagulation in patients with liver disease. J. Am. Coll. Cardiol. 71, 2162-2175 (2018).

24. Kasthuri, R. S., Taubman, M. B. \& Mackman, N. Role of tissue factor in cancer. J. Clin. Oncol. 27, 4834-4838 (2009).

\section{Acknowledgements}

The authors thank the Health and Welfare Data Science Center of the Ministry of Health and Welfare, Taiwan, for maintaining and processing the data, and the Health and Welfare Data Science Center of Tzu Chi University for facilitating data extraction.

\section{Author contributions}

H.J.Y.: Preparation of the manuscript, study conception and design, data extraction, data interpretation; L.P.P.: Study design, data extraction, and statistical analysis; L.A.B.: Study design and data interpretation; H.H.K.: Study conception and design, data interpretation, and critical revision of the manuscript; L.C.H.: Study conception and design, data interpretation, and critical revision of the manuscript.

\section{Competing interests}

The authors declare no competing interests.

\section{Additional information}

Supplementary Information The online version contains supplementary material available at https://doi.org/ 10.1038/s41598-021-89867-0.

Correspondence and requests for materials should be addressed to H.-K.H. or C.-H.L.

Reprints and permissions information is available at www.nature.com/reprints.

Publisher's note Springer Nature remains neutral with regard to jurisdictional claims in published maps and institutional affiliations.

(c) (i) Open Access This article is licensed under a Creative Commons Attribution 4.0 International

License, which permits use, sharing, adaptation, distribution and reproduction in any medium or format, as long as you give appropriate credit to the original author(s) and the source, provide a link to the Creative Commons licence, and indicate if changes were made. The images or other third party material in this article are included in the article's Creative Commons licence, unless indicated otherwise in a credit line to the material. If material is not included in the article's Creative Commons licence and your intended use is not permitted by statutory regulation or exceeds the permitted use, you will need to obtain permission directly from the copyright holder. To view a copy of this licence, visit http://creativecommons.org/licenses/by/4.0/.

(C) The Author(s) 2021 